\title{
On a Class of Variance Balanced Designs
}

\author{
D. K. Ghosh ${ }^{1}$, Shreya Ghosh ${ }^{2} \&$ Narenrda C. Sinohjia ${ }^{3}$ \\ ${ }^{1,3}$ Department of Statistics, Saurashtra University, Gujarat, India \\ ${ }^{2}$ Dep. of Information Security, University of Cumberland, USA \\ Correspondence: D. K. Ghosh, Department of Statistics, Saurashtra University, Rajkot, Gujarat, India.
}

Received: March 19, 2018 Accepted: April 19, 2018 Online Published: April 27, 2018

doi:10.5539/ijsp.v7n3p112 URL: https://doi.org/10.5539/ijsp.v7n3p112

\begin{abstract}
This paper discusses the method of construction of Variance balanced designs using (i) factorial designs and (ii) some incidence matrix. Further the discussion also involves the efficiency criteria of Variance balanced designs. We have also carried out some properties of Variance balanced designs. This study has been supported by suitable examples.
\end{abstract}

Keywords: Non-orthogonal, efficiency factor, balanced, eigen values and parametric relations

\section{Introduction}

Among all the incomplete block design Balanced incomplete Block design is the best incomplete block design because, BIBD is binary, proper, connected, equi-replicated, balanced and non-orthogonal. Hence BIBD is simple and easy to analysis. However it is very difficult to have a BIBD because BIBD exist only when it satisfied the parametric relations (a) $v r=b k$ (b) $\lambda(v-1)=r(k-1)$ and (c) $b \geq v$, and hence in place of BIBD we require another class of incomplete block design which is balanced. This type of incomplete block design is referred as Variance balanced designs. Rao (1958) observed that if the information matrix $C$ of a block design satisfied

$$
\text { C } \theta\left[I_{v}-\frac{1}{v} E_{v v}\right]
$$

where $\theta$ is non zero eigen value of $C$ matrix, $I_{v}$ is an identity matrix of order $v, E_{v v}$ is the matrix with $v$ rows and $v$ columns whose all the elements are unity then such design is called Variance balanced designs. Since BIBD satisfies this property and hence BIBD is also called Variance balanced designs.

Further many investigators studied about the construction and analysis of Variance balanced designs. For an example Roy (1958) carried out the notion of efficiency factor of block design. Chakrabarti (1963) discussed the useful concept of $C$-matrix of design. Pearce(1964) constructed VB Design with varying block sizes. Kageyama (1976), Mukharji and Kageyama (1985) studied Resolvable VB Design with unequal replications. Das and Ghosh (1985) constructed unequal replicated, unequal/equal block sizes VB Designs obtained from BIBD and PBIBD using augmented blocks and treatments. Rao(1958), Hedayat and Federer (1974), Raghvarao (1962) and Puri and Nigam (1977) studied that a design is said to be variance balanced if every normalized estimable linear treatment contrasts can be estimated with same precision. Tyagi (1979), Kulshreshtra et al.(1972), Kageyama (1974, 1976, 1987, 1988) discussed the construction of VB design using BIBD, PBIBD and some incidence matrices.

Khatri (1982) gave a formula to measure over-all-A-Efficiency of VB designs along with method of construction of VB Designs. Gupta and Jones (1983) obtained VB design using BIBD and PBIBD with two associate classes. Hedayat and Federer (1974), Calvin (1986) obtained VB design based on unioning block design. Calvin and Sinha (1989) extended the technique of Calvin (1986) to construct VB designs with more than two distinct block sizes that permit fewer replications. Sinha and Jones (1988) discussed construction of variance balanced designs with unequal block sizes which are more applicable than EB design for the practical point of view as the variance of each treatment contrast is the same in VB design.

Das and Ghosh(1985) defined generalized efficiency balanced (GEB) design which include both VB as well as EB designs. Ghosh (1988), Ghosh and Karmaker (1988), Ghosh and Devecha (1989), Ghosh, Divecha and Kageyama (1991), Ghosh et al. (1992) provided several methods for construction of VB designs. Ghosh and Joshi (1995) constructed VB Design through Triangular design. Agarwal and Kumar (1985) constructed VB design which is associated with group divisible design. Ghosh and Joshi (1991) constructed VB design through GD design. 
Kageyama (1988): recommended the use of non binary VB design when binary VB designs are not available for given values of parameters. Agarwal and Kumar (1986) developed some methods of constructing ternary VB designs with $(v+s)$ treatments $(s \geq 1)$, having blocks of unequal sizes, through block designs with $v$ treatment. Ghosh, Kageyama and Joshi (1993) developed Ternary VB designs using BIB and GD design.

Ghosh and Joshi (1995) also discussed parametric relations of Efficiency Balanced and Variance balanced design. Ghosh (2012) discussed robustness of variance balanced design against the loss of k treatments and one block.

\section{Definition}

A block design is said to be Variance-balanced if it permits the estimation of all estimable normalized treatment contrasts with the same variance, that is,

$$
V\left(\hat{t}_{i}-\hat{t}_{j}\right)=k \sigma^{2}, \quad \forall i \neq j=1,2, \ldots, v .
$$

Variance balance designs can also be further defined in the following way. A connected block design is said to be Variance balanced if and only if all the nonzero eigen values of the $C$ matrix of the block design with multiplicities $(\mathrm{v}-1)$ are equal. We can define variance balanced design also as the following: a connected block design is Variance-balanced if and only if its $C$-matrix has all off its diagonal elements equal and again all of its off diagonal elements are equal, that is,

$$
C=(a-b) I+b j j^{\prime}
$$

It is to be noted that for an equi-replicated, proper, binary, Variance balanced design, the Concurrence matrix Satisfies

$$
N N^{\prime}=(r-\lambda) I_{v}+\lambda j j^{\prime}
$$

Das and Ghosh (1985) suggested that if

$$
\mathrm{C}_{\mathrm{im}}=\mathrm{c} \mathrm{S}_{\mathrm{i}} \mathrm{S}_{\mathrm{m}}
$$

Where $\mathrm{c}$ is a constant, $\mathrm{S}_{\mathrm{i}}$ is the replication size of $\mathrm{i}^{\text {th }}$ treatment and $\mathrm{S}_{\mathrm{m}}$ is the replication size of $\mathrm{m}^{\text {th }}$ treatment, hold true for all $\mathrm{i} \neq \mathrm{m}=1,2, \ldots, \mathrm{v}$. Further they argued that if all $\mathrm{C}_{\mathrm{im}}$ are same then the block design will be called variance balanced designs.

Here we have developed the construction of variance balanced design using symmetrical factorial experiments and by developing some incidence matrices. We have discussed some properties of variance balanced designs which is explained in section 4.

\section{Method of Construction of Variance Balanced Designs.}

\subsection{Variance Balanced Design from Symmetrical Factorial Experiment $2^{n}$ Deleting Control Treatment.}

Theorem 3.1.1 Consider a factorial experiment $2^{\mathrm{n}}$. Deleting the control treatment ( I ) we get a variance balanced designs with parameter $\mathrm{v}=\mathrm{n}, \mathrm{b}=2^{\mathrm{n}}-1, \mathrm{r}=2^{\mathrm{n}-1}, \mathrm{k}=\{1,2, \ldots, \mathrm{n}\}$.

Proof: let the blocks of $2^{\mathrm{n}}$ factorial experiments are

$$
\begin{array}{llllllll}
0 & 0 & 0 & 0 & . & . & . & 1 \\
0 & 0 & 1 & 1 & . & . & . & 1 \\
0 & 1 & 0 & 1 & . & . & . & 1 \\
. & . & . & . & . & . & . & . \\
. & . & . & . & . & . & . & . \\
0 & 1 & 0 & 1 & . & . & . & 1
\end{array}
$$

Deleting the control treatment $\left(\begin{array}{llllll}0 & 0 & 0 & \ldots & \ldots\end{array}\right)$. Call each treatment combinations as one block and factors as treatment. This way we have v treatments and $2^{\mathrm{n}}-1$ blocks. Each treatment combinations has $2^{\mathrm{n}-1}$ "zero's" and “ one's". So design is binary and has $2^{\mathrm{n}-1}$ replications. Further each treatment combinations has either one, two, three, . ., $n$ ' ' 1 '. Hence block sizes is $\{1,2, \ldots, n\}$. So the design is binary and equi replicated.

If we can prove that the incidence matrix satisfy

$$
C=\theta\left[\mathrm{I}_{\mathrm{v}}-\frac{1}{\mathrm{v}} \mathrm{E}_{\mathrm{vv}}\right]
$$

Then the design will be variance balanced, where

$$
\mathrm{C}=\operatorname{diag}\left(\mathrm{r}_{1}, \mathrm{r}_{2}, \ldots, \mathrm{r}_{\mathrm{v}}\right)-\mathrm{NK}^{-1} \mathrm{~N}^{\prime}
$$


Since each row of incidence matrix has half zero and half ' 1 '. So the diagonal elements of $\mathrm{NK}^{-1} \mathrm{~N}^{\text {' is }}$

$$
\begin{gathered}
\frac{\left(\begin{array}{c}
\mathrm{n}-1 \\
0
\end{array}\right)}{1}+\frac{\left(\begin{array}{c}
\mathrm{n}-1 \\
1
\end{array}\right)}{2}+\frac{\left(\begin{array}{c}
\mathrm{n}-1 \\
2
\end{array}\right)}{3}+\frac{\left(\begin{array}{c}
\mathrm{n}-1 \\
3
\end{array}\right)}{4}+\ldots .+\frac{1}{\mathrm{n}} \\
{\left[\begin{array}{cccc}
2^{\mathrm{n}-1} & 0 & \ldots & 0 \\
0 & 2^{\mathrm{n}-1} & \ldots & 0 \\
0 & 0 & \ldots & 2^{\mathrm{n}-1}
\end{array}\right]-\left[\begin{array}{cccc}
\mathrm{B} & \mathrm{A} & \ldots & \mathrm{A} \\
\mathrm{A} & \mathrm{B} & \ldots & \mathrm{A} \\
\vdots & \vdots & \vdots & \vdots \\
\mathrm{A} & \mathrm{A} & \ldots & B
\end{array}\right]}
\end{gathered}
$$

Where $\mathrm{B}=\frac{\left(\begin{array}{c}\mathrm{n}-1 \\ 0\end{array}\right)}{1}+\frac{\left(\begin{array}{c}\mathrm{n}-1 \\ 1\end{array}\right)}{2}+\frac{\left(\begin{array}{c}\mathrm{n}-1 \\ 2\end{array}\right)}{3}+\frac{\left(\begin{array}{c}\mathrm{n}-1 \\ 3\end{array}\right)}{4}+\ldots .+\frac{1}{\mathrm{n}}$

And $\mathrm{A}=\frac{1}{2}+\frac{\mathrm{n}-2}{3}+\frac{\mathrm{n}-3}{4}+\ldots+\frac{\mathrm{n}-(\mathrm{n}-1)}{\mathrm{n}}$

After solving (3.1) we have,

$$
\begin{gathered}
C=\left[\begin{array}{cccc}
2^{\mathrm{n}-1}-\mathrm{B} & -\mathrm{A} & \ldots & -\mathrm{A} \\
-\mathrm{A} & 2^{\mathrm{n}-1}-\mathrm{B} & \ldots & -\mathrm{A} \\
\vdots & \vdots & & \vdots \\
-\mathrm{A} & -\mathrm{A} & \ldots & 2^{\mathrm{n}-1}-\mathrm{B}
\end{array}\right] \\
C=\theta\left[\mathrm{I}_{\mathrm{v}}-\frac{1}{\mathrm{v}} \mathrm{E}_{\mathrm{vv}}\right]
\end{gathered}
$$

Here $\theta$ is non - zero eigen value of $\mathrm{C}$ matrix of block design where $\theta=\left(2^{\mathrm{n}-1}-\mathrm{B}+\mathrm{A}\right)$. This satisfies the criteria of variance balanced design. Hence the design is VB.

\subsubsection{Example 1}

Let $n=3$. So the Symmetrical factorial experiment is $2^{3}$. This factorial design has 8 treatment combinations. The following seven treatment combinations are shown below after deleting the control treatment.

\begin{tabular}{lllllll|l}
0 & 0 & 0 & 1 & 1 & 1 & 1 & 4 \\
0 & 1 & 1 & 0 & 0 & 1 & 1 & 4 \\
1 & 0 & 1 & 0 & 1 & 0 & 1 & 4 \\
\hline 1 & 1 & 2 & 1 & 2 & 2 & 3 &
\end{tabular}

Now from factorial experiment deleting the control treatment, we get a variance balanced design with parameters $\mathrm{v}=\mathrm{n}=3, \mathrm{~b}=2^{\mathrm{n}}-1=7, \mathrm{r}=2^{\mathrm{n}-1}=4, \mathrm{k}=\{1,2, \ldots, 3\}$.

Here B $=\left(\begin{array}{l}2 \\ 0\end{array}\right)+\frac{\left(\begin{array}{l}2 \\ 1\end{array}\right)}{2}+\frac{\left(\begin{array}{l}2 \\ 2\end{array}\right)}{3}=1+\frac{2}{2}+\frac{1}{3}=\frac{6+6+2}{6}=\frac{14}{6}$

$A=\frac{1}{2}+\frac{1}{3}=\frac{5}{6} \quad$ so $N k^{-1} N^{\prime}=\left[\begin{array}{ccc}14 & 5 & 5 \\ 5 & 14 & 5 \\ 5 & 5 & 14\end{array}\right] / 6$

$C$ matrix is

$$
\begin{gathered}
C=\left[\begin{array}{ccr}
4-\frac{14}{6} & -\frac{3}{6} & -\frac{3}{6} \\
-\frac{5}{6} & 4-\frac{14}{6} & -\frac{5}{6} \\
-\frac{5}{6} & -\frac{5}{6} & 4-\frac{14}{6}
\end{array}\right] \\
C=\left[\begin{array}{rrr}
10 & -5 & -5 \\
-5 & 10 & -5 \\
-5 & -5 & 10
\end{array}\right] / 6
\end{gathered}
$$




$$
\begin{gathered}
C=\frac{15}{6}\left[I_{3}-\frac{1}{3} E_{v v}\right] \\
\therefore \theta=\frac{15}{6}
\end{gathered}
$$

3.2 Variance Balanced Design from Symmetrical Factorial Experiment $2^{n}$ Deleting Control Treatment and All Main Effects.

Next we construct variance balanced design from a $2^{\mathrm{n}}$ symmetrical factorial experiments after deleting control treatment and all the main effects. This is discussed in theorem 3.2.1.

Theorem: 3.2.1. If we delete the control treatment and all the main effects and then considering each treatment combination a block we get a variance balanced design with parameters.

$$
\mathrm{v}=\mathrm{n}, \mathrm{b}=2^{\mathrm{n}}-1-\left(\begin{array}{l}
\mathrm{n} \\
1
\end{array}\right), \mathrm{r}=2^{\mathrm{n}-1}-1, \mathrm{k}=(2,3, \ldots, \mathrm{n}) .
$$

Proof: consider a $2^{\mathrm{n}}$ treatment combination. Delete its control treatment and all main effects. Consider each treatment combination compose of $\left[\begin{array}{lllll}0 & 1 & 0 & 1 & \ldots . .\end{array}\right]$ as one block. This way we have $\left(2^{\mathrm{n}}-1-\mathrm{n}\right)$ column with $\mathrm{v}$ treatments as the incidence matrix of a block design. This incidence matrix has all elements as zero or 1 only. So the design is binary.

Now each treatment is repeated $\left(2^{\mathrm{n}-1}-1\right)$ times so design is equireplicated and unequal block of $\{2,3, \ldots, \mathrm{n}\}$

Let the incidence matrix of the block design is given by

$$
N=\left[\begin{array}{cccccc}
0 & 1 & 1 & 1 & \vdots & 1 \\
1 & 0 & 0 & 1 & \vdots & 1 \\
1 & 1 & 1 & 0 & \vdots & 1 \\
\vdots & \vdots & \vdots & \vdots & \vdots & 1 \\
0 & 0 & 1 & 1 & \vdots & 1
\end{array}\right]
$$

If we can prove that the $C$ matrix of the block design satisfy $C=\theta\left[I_{v}-\frac{1}{v} E_{V v}\right]$

Then design is variance balanced where $\theta$ is non - zero eigen value of $\mathrm{C}$ - matrix, Where

$$
\mathrm{C}=\operatorname{diag}\left(\mathrm{r}_{1}, \mathrm{r}_{2}, \ldots, \mathrm{r}_{\mathrm{v}}\right)-\mathrm{NK}^{-1} \mathrm{~N},
$$

Further each row of incidence matrix $N$ contain ' 1 ' $\left(2^{n-1}-1\right)$ times . So the diagonal elements of the $N K^{-1} N^{\prime}$ matrix is

$$
\mathrm{S}=\frac{\left(\begin{array}{c}
\mathrm{n}-1 \\
1
\end{array}\right)}{2}+\frac{\left(\begin{array}{c}
\mathrm{n}-1 \\
2
\end{array}\right)}{3}+\frac{\left(\begin{array}{c}
\mathrm{n}-1 \\
3
\end{array}\right)}{4}+\ldots .+\frac{1}{\mathrm{n}}
$$

Again all of off diagonal elements of $\mathrm{NK}^{-1} \mathrm{~N}^{\prime}$ are

$$
\mathrm{R}=\frac{1}{2}+\frac{\mathrm{n}-2}{3}+\frac{\mathrm{n}-3}{4}+\ldots+\frac{\mathrm{n}-(\mathrm{n}-1)}{\mathrm{n}} \quad \text { and } \mathrm{r}=\left(2^{\mathrm{n}-1}-1\right)
$$

after solving (3.3) we have

$$
\begin{gathered}
\mathrm{C}=\left[\begin{array}{cccc}
2^{\mathrm{n}-1} & 0 & \ldots & 0 \\
0 & 2^{\mathrm{n}-1} & \ldots & 0 \\
0 & 0 & \ldots 2^{\mathrm{n}-1}
\end{array}\right]-\left[\begin{array}{cccc}
\mathrm{S} & \mathrm{R} & \ldots & \mathrm{R} \\
\mathrm{R} & \mathrm{S} & \ldots & \mathrm{R} \\
\vdots & \vdots & \vdots & \vdots \\
\mathrm{R} & \mathrm{R} & \ldots & \mathrm{S}
\end{array}\right] \\
\mathrm{C}=\left[\begin{array}{cccc}
2^{\mathrm{n}-1}-\mathrm{S} & -\mathrm{R} & \ldots & -\mathrm{R} \\
-\mathrm{R} & 2^{\mathrm{n}-1}-\mathrm{S} & \ldots & -\mathrm{R} \\
\vdots & \vdots & \vdots \\
-\mathrm{R} & -\mathrm{R} & \ldots & 2^{\mathrm{n}-1}-\mathrm{S}
\end{array}\right] \\
C=\theta\left[\mathrm{I}_{\mathrm{v}}-\frac{1}{\mathrm{v}} \mathrm{E}_{\mathrm{vv}}\right]
\end{gathered}
$$


Here $\theta$ is non - zero eigen value of $C$ matrix of block design, where $\theta=\left(2^{n-1}-1-S+R\right)$

Since (3.5) satisfy the criteria of variance balance design and hence the existing design is a variance balanced design.

3.2.2.Example 2. Consider a $2^{3}$ factorial experiment. This has eight treatment combinations. Delete all main effects and control treatment. In this way we have four treatment combinations. Call each treatment combination as a block of VB design. Thus we have four blocks with 3 treatments. Further consider this as a incidence matrix of a block design. This is shown below

$$
\mathrm{N}=\begin{array}{lllll}
0 & 1 & 1 & 1 & 3 \\
1 & 0 & 1 & 1 & 3 \\
1 & 1 & 0 & 1 & 3 \\
\hline 2 & 2 & 2 & 3
\end{array}
$$

Using this method we get another variance balanced design with smaller number of replication and also with less number of block size.

$$
\begin{aligned}
& \mathrm{NK}^{-1}=\left[\begin{array}{llll}
0 & 1 & 1 & 1 \\
1 & 0 & 1 & 1 \\
1 & 1 & 0 & 1
\end{array}\right]\left[\begin{array}{cccc}
1 / 2 & 0 & 0 & 0 \\
0 & 1 / 2 & 0 & 0 \\
0 & 0 & 1 / 2 & 0 \\
0 & 0 & 0 & 1 / 3
\end{array}\right]=\left[\begin{array}{cccc}
0 & 1 / 2 & 1 / 2 & 1 / 3 \\
1 / 2 & 0 & 1 / 2 & 1 / 3 \\
1 / 2 & 1 / 2 & 0 & 1 / 3
\end{array}\right] \\
& \mathrm{NK}^{-1} \mathrm{~N}^{\prime}=\left[\begin{array}{cccc}
0 & 1 / 2 & 1 / 2 & 1 / 3 \\
1 / 2 & 0 & 1 / 2 & 1 / 3 \\
1 / 2 & 1 / 2 & 0 & 1 / 3
\end{array}\right]\left[\begin{array}{ccc}
0 & 1 & 1 \\
1 & 0 & 1 \\
1 & 1 & 0 \\
1 & 1 & 1
\end{array}\right]=\frac{\left[\begin{array}{lll}
8 & 5 & 5 \\
5 & 8 & 5 \\
5 & 5 & 8
\end{array}\right]}{6} \\
& C=\left[\begin{array}{lll}
3 & 0 & 0 \\
0 & 3 & 0 \\
0 & 0 & 3
\end{array}\right]-\frac{\left[\begin{array}{lll}
8 & 5 & 5 \\
5 & 8 & 5 \\
5 & 5 & 8
\end{array}\right]}{6} \\
& \mathrm{C}=\frac{\left[\begin{array}{rrr}
10 & -5 & -5 \\
-5 & 10 & -5 \\
-5 & -5 & 10
\end{array}\right]}{6} \\
& C=\frac{5}{6}\left[\begin{array}{ccc}
2 & -1 & -1 \\
-1 & 2 & -1 \\
-1 & -1 & 2
\end{array}\right]=\frac{5}{6}\left[3 \mathrm{I}_{3}-\mathrm{E}_{33}\right] \\
& \mathrm{C}=\frac{15}{6}\left[\mathrm{I}_{3}-\frac{1}{3} \mathrm{E}_{33}\right]
\end{aligned}
$$

So the design is variance balanced.

\section{Properties of Variance Balanced Designs}

Theorem 4.1: For a Variance balanced design with parameters $v, b, r_{i}, k_{j}$ and $\lambda$, it is observed that $\theta=v c_{i m}$ always holds true, where $\theta$ is non zero eigen values with multiplicity $(v-1)$, and $\quad c_{i m}=\sum_{j=1}^{b} \frac{n_{i j} n_{m j}}{n_{. j}}$.

Proof : Let us consider a VB designs with parameters $v, b, r_{i}, k_{j}$ and $\lambda$, where $\lambda$ denotes number of block in which a pair of treatment occurs. The reduced normal equation for a block design is given as

$$
c_{i i} t_{i}+c_{i m} t_{m}=Q_{i}
$$




$$
\text { where } c_{i i}=\sum_{j=1}^{b} n_{i .}-\sum_{j} \frac{n_{i j^{2}}}{n_{. j}}, \quad c_{i m}=\sum_{j=1}^{b} \frac{n_{i j} n_{m j}}{n_{. j}}, \quad \forall i \neq m
$$

Suppose design is binary, equiblock size, equireplicated and $\sum n_{i j} n_{m j}=\lambda$ then $c_{i m}=\frac{\lambda}{k}$, we know for such design, the non zero eigen value of $C$ matrix of design $d$ is $\theta=\frac{\lambda v}{k}$ with multiplicity $(v-1)$, hence $\theta=v \cdot c_{i m}$.

Consider a BIBD with parameters $v=4, v=6, r=3, k=2, \lambda=1$

$$
\begin{gathered}
C=\left[\begin{array}{rrrr}
3 & -1 & -1 & -1 \\
-1 & 3 & -1 & -1 \\
-1 & -1 & 3 & -1 \\
-1 & -1 & -1 & 3
\end{array}\right] / 2 \\
=\left[4 I_{v}-j_{v} j_{v^{\prime}}\right] / 2 \\
=\frac{4}{2}\left[I_{v}-\frac{1}{4} j_{v} j_{v^{\prime}}\right]=\frac{4}{2}\left[I_{v}-\frac{1}{v} E_{v v}\right] \\
\therefore \theta=\frac{4}{2}
\end{gathered}
$$

$\theta=v \cdot c_{i m}=4 \cdot \frac{1}{2}$, where $\mathrm{C}_{\mathrm{im}}$ is obtained from

$$
c_{i m}=\sum \frac{n_{i j} n_{m j}}{n_{. j}}=\frac{1}{2}
$$

For example 2, $v=3$ and $c_{i m}=5 / 6$ hence $\theta=v \cdot c_{i m}=3 \cdot \frac{5}{6}=\frac{15}{6}$

\section{Construction of Variance Balanced Designs Using Some Incidence Matrices}

So far we discussed Variance balanced designs of equi-replicated and Unequal block Sizes. In fact, the following four types of Variance balanced design Can exists namely

(a) equi-replicated and equi-block sizes (Proper) VB design

(b) equi-replicated and Unequal block sizes VB design

(c) Unequal replication and equi-block sizes VB design

(d) Unequal replications and Unequal block sizes VB designs

Now we discuss the Construction of Variance balanced designs with Unequal replication Sizes and Unequal block sizes. This is shown in Theorem 5.1

5.1 Theorem 5.1 : Let $I_{n}$ denotes the identity matrix of order $n, j_{m}$ is a Column Vector of one, $j_{m}^{\prime}$ is the transpose of $j_{m}$ and $0_{n}$ is the row vectors having all elements zero, Now an incidence matrix $N$ defined as

$$
N=\left[\begin{array}{ll}
j_{n}^{\prime} & 0_{1 \times \frac{n}{2}} \\
I_{n} & j_{n} j_{\frac{n}{2}}^{\prime}
\end{array}\right]
$$

gives the incidence matrix of a Variance balanced designs, where $n$ is even, with parameters

$v=n+1, \quad b=n+\frac{n}{2}, \quad r=\left\{n, 1+\frac{n}{2}\right\} \quad$ and $k=\{2, n\}$

Proof : Proof is obvious.

5.2 Example 3 . Let $n=4$ So the incidence matrix $N$ using Theorem 5.1 Can be written as 


$$
N=\left[\begin{array}{llllll}
1 & 1 & 1 & 1 & 0 & 0 \\
1 & 0 & 0 & 0 & 1 & 1 \\
0 & 1 & 0 & 0 & 1 & 1 \\
0 & 0 & 1 & 0 & 1 & 1 \\
0 & 0 & 0 & 1 & 1 & 1
\end{array}\right]
$$

Here $v=5, b=6, r=\{4,3\}, k=\{2,4\}$

$$
\begin{gathered}
N K^{-1} N^{\prime}=\left[\begin{array}{lllll}
8 & 2 & 2 & 2 & 2 \\
2 & 4 & 2 & 2 & 2 \\
2 & 2 & 4 & 2 & 2 \\
2 & 2 & 2 & 4 & 2 \\
2 & 2 & 2 & 2 & 4
\end{array}\right] / 4 \\
C=\left[\begin{array}{lllll}
4 & 0 & 0 & 0 & 0 \\
0 & 3 & 0 & 0 & 0 \\
0 & 0 & 3 & 0 & 0 \\
0 & 0 & 0 & 3 & 0 \\
0 & 0 & 0 & 0 & 3
\end{array}\right]-\left[\begin{array}{lllll}
8 & 2 & 2 & 2 & 2 \\
2 & 4 & 2 & 2 & 2 \\
2 & 2 & 4 & 2 & 2 \\
2 & 2 & 2 & 4 & 2 \\
2 & 2 & 2 & 2 & 4
\end{array}\right] / 4
\end{gathered}
$$

or

$$
\begin{gathered}
C=\left[\begin{array}{rrrrr}
8 & -2 & -2 & -2 & -2 \\
-2 & 8 & -2 & -2 & -2 \\
-2 & -2 & 8 & -2 & -2 \\
-2 & -2 & -2 & 8 & -2 \\
-2 & -2 & -2 & -2 & 8
\end{array}\right] / 4 \\
C=\frac{10}{4}\left[I_{5}-\frac{1}{5} E_{55}\right] \\
\therefore \theta=\frac{10}{4}=\frac{5}{2}
\end{gathered}
$$

Now Using the Theorem 4.1, $\theta=v \cdot c_{i m}$, So we have $\theta=5 \cdot \frac{1}{2}=5 / 2$, which Satisfies the theorem.

Remark. Equi-replicated, equi-block sizes (Proper) binary Variance balanced design is infect, balanced incomplete block designs.

For an example $v=4, b=6, r=3, k=2, \lambda=1$ is a BIBD whose $C$-matrix is

$$
\left[\begin{array}{rrrr}
3 & -1 & -1 & -1 \\
-1 & 3 & -1 & -1 \\
-1 & -1 & 3 & -1 \\
-1 & -1 & -1 & 3
\end{array}\right] / 2
$$

Here $\theta=v \cdot c_{i m}=4 \times \frac{1}{2}=2$ 


\section{Analysis of Variance Balance Designs}

Consider Theorem 3.1. Let $t_{i}(i=1, \ldots, v)$ be treatment effects of $i^{\text {th }}$ treatments. The Solution of estimate of treatment effect $\hat{t}_{i}=\frac{1}{\theta} Q_{i}=\left(\frac{1}{2^{n-1}-B+A}\right) Q_{i}$. Variance of treatment Contrasts of $\hat{t}_{i}$ and $\hat{t}_{j}$ is

$V\left(\hat{t}_{i}-\hat{t}_{j}\right)=\frac{2}{\theta} \sigma^{2}=\left(\frac{1}{2^{n-1}-B+A}\right) \sigma^{2}$, where $\sigma^{2}$ denotes error mean sum of square.

\subsection{Efficiency Factor}

Efficiency factor of variance balanced designs compare to completely randomized design is given by

$$
E=\frac{V\left(\hat{t}_{i}-\hat{t}_{j}\right)_{C R D}}{V\left(\hat{t}_{i}-\hat{t}_{j}\right)_{V B}}
$$

Now we consider the example 1 , where $\theta=\frac{15}{8}$, so $\hat{t}_{i}=\frac{6}{15} Q_{i}$ and hence $V\left(\hat{t}_{i}-\hat{t}_{j}\right)=\frac{2}{\theta} \sigma^{2}$

$$
=\frac{2}{15 / 6} \sigma^{2}=\frac{12}{15} \sigma^{2}
$$

Hence, $E=\frac{\frac{2}{4} \sigma^{2}}{\frac{12}{15} \sigma^{2}}=\frac{30}{48}=0.62$

\section{References}

Agarwal, G. G., \& Kumar, S. (1984). A note on construction of variance balanced designs associated with GD designs. Calcutta Statist. Assoc. Bull. 33, 187C190.

Agarwal, G. G., \& Kumar, S. (1985). A note on construction of variance balanced designs. Jour.Ind. Soci. Agri. Stati. $37(2), 181 \mathrm{C} 183$.

Agarwal, G. G., \& Kumar, S. (1986). Construction of balanced ternary designs. Austral. J. Statist. 28, $251 \mathrm{C} 255$. https://doi.org/10.1111/j.1467-842X.1986.tb00605.x

Bose, R. C., \& Nair, K. R. (1939). Partially balanced incomplete block design. Snkhya, 4, 337C372.

Calvin, J. A. (1986). A new class of variance balanced designs. J. Statist.Plann. Inference, 14, $251 \mathrm{C} 254$. https://doi.org/10.1016/0378-3758(86)90162-X

Calvin, J. A., \& Sinha, K. (1989). A method of constructing variance balanced designs. J. Statist. Plann. Int. $23,127 C 131$. https://doi.org/10.1016/0378-3758(89)90045-1

Chakrabarti, M. C. (1963). On C-matrix in design of experiments. J. Indian. Stat. Assoc. 1, 8C23.

Das, M. N., \& Ghosh, D. K. (1985). Balancing incomplete block designs. Sankhya, B.47, 67C77.

Dey, A. (1970). On construction of balanced n-ary block designs. Ann.Inst.Statist.Math. 22, 389C393. https://doi.org/10.1007/BF02506352

Ghosh, D. K. (1988). A series of generalized efficiency balanced designs. Gujrat Statistical Review, $15(1), 33$ C38.

Ghosh, D. K., \& Divecha, J. (1989). Construction of variance balanced designs. TEC.R.No.2, Dept. of Maths. And Stat., Saurashtra Uni. $1 \mathrm{C} 13$

Ghosh, D. K., Divecha, J., \& Kageyama, S. (1991). Equireplicate variance balanced designs from group divisible designs. J.Japan. Statist. Soc. 21(1), 205C209. https://doi.org/10.1111/j.1467-842X.1988.tb00610.x

Ghosh, D. K., \& Joshi, K. (1991). Note on construction of variance balanced designs through group divisible designs. Utilitas Math. 39, 249C253.

Ghosh, D. K., \& Joshi, K. (1995). Construction of variance balanced designs through Triangular PBIB dsesigns. Calcutta Statist. Asson. Bull. 45,177- 178, 111- 118.

Ghosh, D. K., Joshi, K., \& Kageyama, S. (1993). Ternary variance balanced designs through BIB and GD designs. J. Japan. Statist. Soc. 23(1), 75C81. 
Ghosh, D. K., \& Karmoker, P. K. (1988). Some series of efficiency balanced designs. Austral. J. Statist. 30(1), 47C51.

Ghosh, D. K. (2012). Robustness of Variance balanced designs against the loss of one block. Indian Jour. of Statistics and applications, $1,17 \mathrm{C} 30$.

Gupta, S. C., \& Jones, B. (1983). Equireplicated balanced block designs with unequal block sizes. Biometrika, 70, 443C440. https://doi.org/10.1093/biomet/70.2.433

Hedayat, A., \& Federr, W. T. (1974). Pairwise and variance balanced incomplete block design. Ann.inst. Statist. Math. 26, 331C338. https://doi.org/10.1007/BF02479828

John, P. W. M. (1964). Balanced designs with unequal number of replications. Ann. Math. Statist. 35, 897C899. https://doi.org/10.1214/aoms/1177703597

Jones, B., sinha, K., \& Kageyama, S. (1987). Further equireplicated variance balanced designs with unequal block sizes. Utilitas Math. 32, 5C10.

Kageyama, S. (1974). Reduction of associate classes for block designs and related combinatorial arrangements. Hiroshima Math. J. 4, 527C618.

Kageyama, S. (1976). Construction of balanced block designs. Utilitas Math. 9, 209C229.

Kageyama, S. (1988). Existence of variance balanced binary designs with fewer experimental units. Stat.Prob.Letters, 7, 27C28. https://doi.org/10.1016/0167-7152(88)90083-1

Khatri, C. G. (1982). A note on variance balanced designs. J. Statist. Plann. Inter. 6, $173 \mathrm{C} 177$. https://doi.org/10.1016/0378-3758(82)90086-6

Kulshreshtha, A. C., Dey, A., \& Saha, G. M. (1972). Balanced designs with unequal replications and unequal block sizes. Ann. Math. Statist., 43, 1342C1345. https://doi.org/10.1214/aoms/1177692487

Mukerjee, R., \& Kageyama, S. (1985). On resolvable and affine resolvable variance balanced designs. Biometrika, 72 , 165C172. https://doi.org/10.1093/biomet/72.1.165

Pal, S., \& Pal, S. (1988). Nonproper variance balanced designs and optimality. Commun. Statist. Theor. Meth. 17, 1685C1695. https://doi.org/10.1080/03610928808829706

Pearce, S. C. (1964). Experimenting with block of natural sizes. Biometrices, 20, 699C706. https://doi.org/10.2307/2528123

Puri, P. D., \& Nigam, A. K. (1977). Balanced block designs. Commun. Statist. Theor. Meth., A(12), $1171 \mathrm{C} 1179$. https://doi.org/10.1080/03610927708827560

Raghavrao, D. (1962). On balanced unequal block designs. Biometrika, 49, 561C562. https://doi.org/10.1093/biomet/49.3-4.561

Rao, V. R. (1958). A note on balanced designs. Ann. Math. Statist. 29, 290 C294. https://doi.org/10.1214/aoms/1177706729

Roy. J. (1958). On the efficiency factor of block designs. Sankhya, 19, 181C188.

Sinha, K. (1988). A note on equireplicated balanced block designs from BIB designs. J.Ind. Soc. Agric. Statist. 42, $150 \mathrm{C} 153$.

Sinha, K. (1989). Some new equireplicated balanced block designs. Statist.Prob.Lett. 8, 89. https://doi.org/10.1016/0167-7152(89)90089-8

Sinha, K., \& Jones, B. (1988). Further equireplicated balanced block designs with unequal block sizes. Statist. Prob. Lett., 6, 229C330.

Tyagi, B. N. (1979). On a class of variance balanced block designs. J. Statist. Plann. Infor., 3, 333-336. https://doi.org/10.1016/0378-3758(79)90029-6

Yates, F. (1936a). A new method of arranging variety trials involving a large number of varieties. J.Agri.Sci. 26, $424 C 455$.

\section{Copyrights}

Copyright for this article is retained by the author(s), with first publication rights granted to the journal.

This is an open-access article distributed under the terms and conditions of the Creative Commons Attribution license (http://creativecommons.org/licenses/by/4.0/). 